\title{
Lancement d'un logiciel de formation à la radioprotection
}

\section{J. PENNEROUX*}

(Manuscrit reçu le 20 avril 1994)

\begin{abstract}
RÉSUMÉ Un outil de formation multimédia "Radioprotection", réalisé par le CEA, CIS-bio, COGEMA et la société GENESIE, vient d'être commercialisé. Il est constitué d'une base de données à laquelle il peut être associée de 2 à 7 études de cas intéressant le domaine industriel et le domaine médical.
\end{abstract}

ABSTRACT A multimedia training tool entitled "Radioprotection" (Radiation protection) and developed by CEA, CIS-Bio, COGEMA and GENESIE is just available. It is made up of a data basis to which studies (from 2 to 7 ) of cases in the industrial or medical fields can be associated

Le CEA, Cis-bio international, Cogema et Génésie Conception médiatique viennent de réaliser un outil de formation interactif sur la radioprotection, en collaboration avec le CNRS, l'INRS, l'INSERM et l'institut Pasteur, et avec le soutien du ministère de l'enseignement supérieur et de la recherche.

Le logiciel "Radioprotection"(1), programme d'information et de formation interactif, propose une approche pratique de la radioprotection ; il est destiné à toutes les catégories de personnel (laboratoires, industries, secteurs médicaux) pouvant être amenées à manipuler des sources de rayonnement.

Cet outil de formation multimédia(2) est une base de données à laquelle il peut être associé de 2 à 7 études de cas. Elle aborde, à partir de 200 fiches (soit environ 1000 écrans), les thèmes suivants : structure de la matière, radioactivité, interaction rayonnements/matière, source de rayonnements, grandeurs et unités, exposition aux rayonnements, effet des rayonnements sur l'homme, réglementation, protection contre l'exposition interne, appareils de mesure, incidents, radioéléments, appareils et techniques, déchets, adresses utiles, bibliographie.

\footnotetext{
* Commissariat à l'énergie atomique, Direction centrale de la sécurité (DCS/SHST), 29-33, rue de la Fédération, 75752 Paris Cedex 15.

(1) Tous renseignements concernant ce logiciel peuvent être fournis par la Société GENESIE, 4, rue du Faubourg Montmartre, 75009 Paris. Tél. : (1) 42.46.88.10. Fax : 42.46.50.75.

(2) Le système de formation multimédia est une technique d'enseignement, basée sur un dialogue interactif entre l'utilisateur et l'ordinateur.
} 
Les 7 études de cas, sous forme de modules indépendants, sont spécifiques à des secteurs professionnels : laboratoires, industriels, applications médicales. Elles portent plus particulièrement sur :

- sources scellées,

- sources non scellées,

- gammagraphie (industrie),

- installation nucléaire de base (industrie),

- curiethérapie, radiothérapie métabolique,

- scintigraphie,

- radiologie d'exploration et d'intervention chirurgicale.

L'utilisateur peut :

- consulter la base de données dans son ensemble,

- accéder à une sélection de fiches adaptées à sa fonction, fiches),

- accéder à la base par mots clés (à chaque mot clé correspond une liste de

- sélectionner les fiches qui l'intéressent et construire son propre parcours, mémorisable sur disque dur ou disquette,

- chaque entreprise (service formation ou sécurité) peut en "mode expert" (accès réservé) créer de nouveaux profils et y associer les fiches de son choix, ainsi que créer de nouvelles fiches qui viendront enrichir la base de données.

L'objectif général des études de cas est d'être capable d'analyser les risques et de choisir les moyens de prévention et de protection les mieux adaptés. Dans chaque étude de cas, l'utilisateur est placé dans une situation professionnelle où se succèdent des apports d'informations et des exercices à partir de cette situation.

Types d'exercices :

- question à choix unique,

- question à choix multiple,

- question à choix croisé,

- classement des réponses par ordre :

- chronologique (dans le cas des modes opératoires),

- croissant ou décroissant (évaluation du risque associé à des conditions de travail),

- déterminer dans une animation les erreurs opératoires ou comportementales,

- question ouverte. 